\title{
Navigation and Control of Underwater Tracked Vehicle Using Ultrashort Baseline and Ring Laser Gyro Sensors
}

\author{
Dae-Hyeong Ji, ${ }^{1}$ Hyeung-Sik Choi,${ }^{*}$ Mai The Vu, ${ }^{2}$ \\ Ngoc-Duc Nguyen, ${ }^{1}$ and Seo-Kang Kim $^{1}$ \\ ${ }^{1}$ Department of Mechanical Engineering, Korea Maritime and Ocean University, \\ 727, Taejong-ro, Yeongdo-gu, Busan 49112, Republic of Korea \\ ${ }^{2}$ Ocean Science and Technology School, Korea Institute of Ocean Science and Technology-Korea Maritime \\ and Ocean University, 727, Taejong-ro, Yeongdo-gu, Busan 49112, Republic of Korea
}

(Received April 17, 2018; accepted March 20, 2019)

Keywords: underwater navigation system, ring laser gyro (RLG), ultrashort baseline (USBL), integrated navigation system, Kalman filter

In this paper, we present a study on a navigation and control system including a new navigation algorithm for an unmanned underwater track vehicle (UTV). Generally, in underwater navigation, a Doppler velocity $\log$ (DVL) is used to measure the velocity of underwater vehicles. However, the UTV cannot use the DVL since it is operated at the bottom of the seafloor. Therefore, in this study, we developed a hybrid navigation system (HNS) consisting of an inertial measurement unit (IMU), a ring laser gyro (RLG) sensor, and an ultrashort baseline (USBL) sensor for underwater vehicles. The system states of the UTV were estimated using the navigation model and the navigation system composed of the RLG and USBL sensors with an extended Kalman filter (EKF). In the navigation system, the attitude control was designed using the RLG sensor, and the position of the UTV relative to that of the global positioning system (GPS) of the surface ship was estimated using the USBL sensor. A new position and orientation estimation algorithm called the compensating hybrid navigation algorithm (CHNA) was developed using the HNS. The developed CHNA was based on a Kalman filter to more accurately estimate the location of a real underwater trenching vehicle called URI-R using hybrid RLG and USBL sensors by filtering out noises and disturbances. To apply the CHNA to the underwater robotics its rocker (URI-R), we developed a small UTV as a test bed and applied the CHNA to the UTV. Then, we applied the CHNA to the URI-R and verified the superior performance of trajectory tracking through a real underwater test.

\section{Introduction}

Korea Institute of Ocean Science and Technology is developing track-based underwater construction robots named underwater robotics its rocker (URI-R) such as that shown in Fig. 1. The purpose of developing the URI-R is to carry out the task of burying cables along the

*Corresponding author: e-mail: hchoi@kmou.ac.kr https://doi.org/10.18494/SAM.2019.2278 


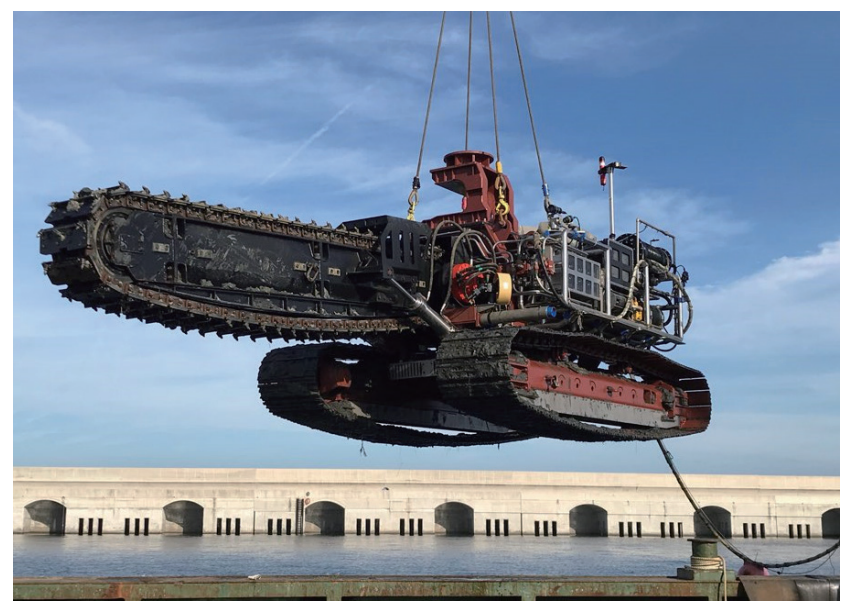

Fig. 1. (Color online) Tack-based underwater construction robot under development.

desired path in water. In underwater navigation, the development of the URI-R such as a remotely operated vehicle (ROV) is essential. ${ }^{(1)}$

In general, the sensors used for underwater navigation include an inertial measurement unit (IMU), a global positioning system (GPS), a Doppler velocity log (DVL), an ultrashort baseline (USBL), and a depth sensor. The equipment currently under development will be developed in the form of track-based construction equipment and used under a turbid environment due to the nature of underwater construction. If an impact disturbance occurs during trenching, the acceleration signal measured as the motion of the equipment may be evaluated to be relatively small and disturbed, such that the navigation operation using the sensor may not be performed appropriately. Also, the DVL and USBL cannot be used if the surrounding turbidity is very high. ${ }^{(2)}$ Therefore, a method of reducing the position error by underwater navigation using the IMU should be studied.

The IMU can be used as the main sensor of an integral navigation system (INS), ${ }^{(3)}$ and the USBL is an underwater acoustic sensor that recognizes the absolute coordinates in water, similarly to the GPS on land. Bijker and Steyn used an extended Kalman filter (EKF) twice to combine a GPS and an INS (loosely coupled system). ${ }^{(4)}$ The reason for using the EKF twice is that the first EKF estimates the attitude of the hull and the second EKF is used to estimate the velocity and position of the hull. Wendel and Trommer used a tightly coupled system consisting of a GPS and an INS because of the pseudo-range information of a GPS in strong coupling, and it is confirmed that the data is processed and the loosely coupled system is excellent. ${ }^{(5)}$ Ridao et al. and Ribas et al. studied DVL fusion considering the time delay of the USBL measurement signal and applied it to the underwater navigation of autonomous underwater vehicles (AUVs). ${ }^{(6,7)}$ Lee et al. proposed a complex navigation algorithm that combines the distance information with the inertial navigation algorithm. ${ }^{(8)}$ In 2015, a fusion of USBL, DVL, and IMU signals was performed. ${ }^{(9)}$ Jung et al. conducted a study to monitor the position of a ROV in a USV by combining an IMU and a USBL. ${ }^{(10)}$ In particular, when a USBL cannot be used owing to high turbidity, a hybrid integrated navigation system composed of a ring laser gyro (RLG) or IMU with an EKF for the accurate tracking of the trajectory is utilized. 
Section 2 of this paper describes the appearance and specifications of the URI-R and underwater track vehicle (UTV) under study. In Sect. 3, the construction of a hybrid navigation system (HNS) using an IMU, an RLG, and a USBL and the study of compensating hybrid navigation algorithm (CHNA) applied to the HNS are discussed. In Sect. 4, the navigation system developed and experimented on land and sea is presented, and Sect. 5 shows the conclusions of this study.

\section{System Overview}

\subsection{URI-R}

The URI-R is a track-based underwater work robot that performs underwater submarine cable/pipeline submergence and subsea underwater construction work on the seabed. Currently, the URI-R is under development, and various driving and navigation experiments are under way. As shown in Table 1, the developed URI-R was designed to have a length of $5800 \mathrm{~mm}$, an endpoint distance of $3495 \mathrm{~mm}$ on both sides of the infinite orbit, and a range of $2595 \mathrm{~mm}$ from the bottom to the top. ${ }^{(11)}$ A total of two hydraulic motors control each endless track individually and can be turned forward/backward and left/right depending on the control method. In the paper, the application of the developed HNS and CHNA to the URI-R is presented.

\subsection{UTV}

We designed and manufactured a small UTV to apply the HNS to the URI-R and CHNA to navigate it along the desired trajectory. For this, the UTV shown in Fig. 2 was designed and constructed as a test bed in a reduced of URI-R form and equipped with infinite tracks for movement on the land and seafloor. As shown in Table 2, the external shape was designed to have a length of $1200 \mathrm{~mm}$, the endpoint distance of both endless tracks was $700 \mathrm{~mm}$, and the distance from the bottom to the top was $800 \mathrm{~mm}$. A total of two electric motors control each infinite track independently and can be rotated forward, backward, and left or right depending on the control method. The navigation sensor is also shown in Fig. 3. As shown in Fig. 3, we constructed a compact HNS that combines an RLG, a magnetic compass, and a USBL. We applied the CHNA to the UTV equipped with the developed HNS to operate the UTV along the desired path accurately in various underwater environments.

Table 1

Specifications of URI-R.

\begin{tabular}{lcc}
\hline Index & Value & Units \\
\hline Length & 5800 & $\mathrm{~mm}$ \\
Width & 3495 & $\mathrm{~mm}$ \\
Height & 2595 & $\mathrm{~mm}$ \\
Weight & 35 & $\mathrm{t}$ \\
Contact length of track & 400 & $\mathrm{~mm}$ \\
Track width & 900 & $\mathrm{~mm}$ \\
Standard components & IMU, Depth, USBL, GPS, TCM, altimeter, camera (analog/HD), \\
& image sonar, oil hydraulic motor, trenching tool \\
\hline
\end{tabular}



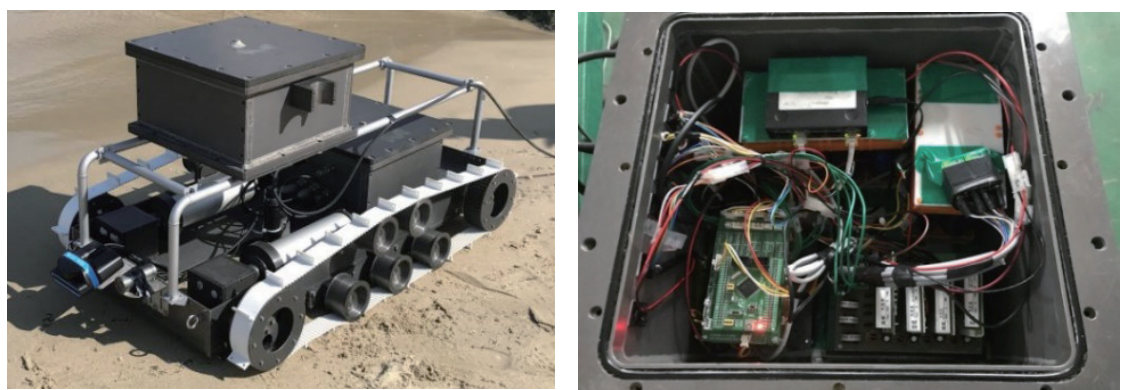

Fig. 2. (Color online) Developed UTV.

Table 2

Specifications of UTV.

\begin{tabular}{lrc}
\hline Index & Value & Units \\
\hline Length & 1200 & $\mathrm{~mm}$ \\
Width & 700 & $\mathrm{~mm}$ \\
Height & 800 & $\mathrm{~mm}$ \\
Weight (dry) & 100 & $\mathrm{~kg}$ \\
Weight (water) & 5 & $\mathrm{~kg}$
\end{tabular}

Standard components IMU, depth, USBL, GPS, TCM, altimeter, camera, forward looking sonar, DC motor
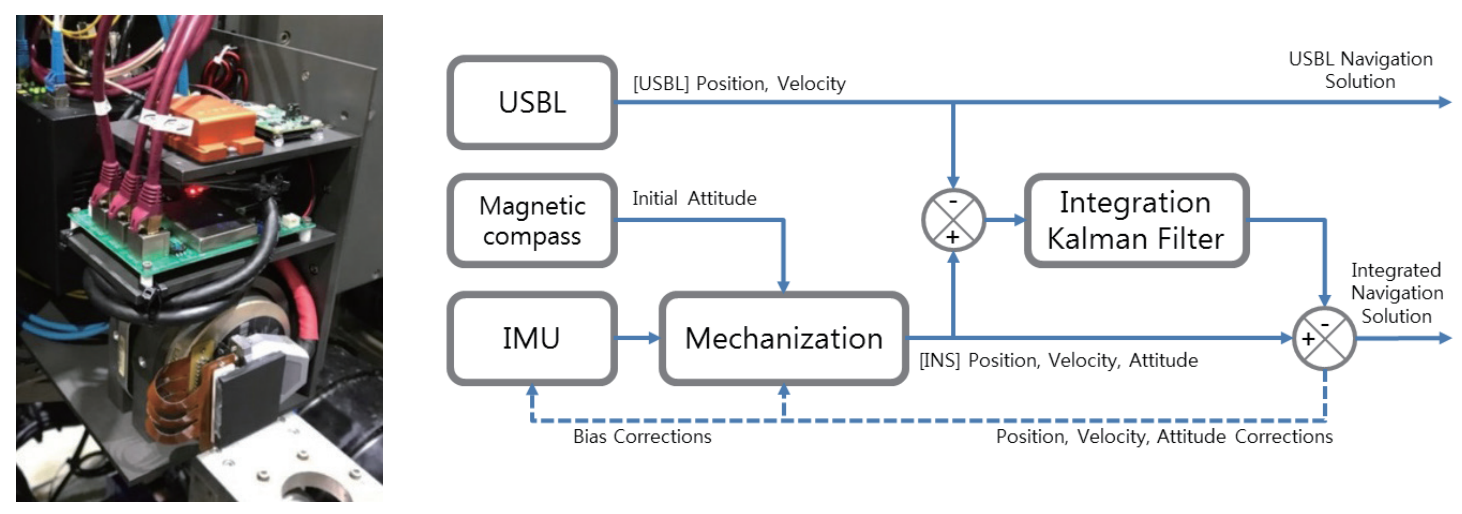

Fig. 3. (Color online) Picture and structure of HNS.

\section{Compensating HNA}

The IMU, which is the primary sensor of a strap-down INS, outputs the acceleration and angular velocity in a body-fixed coordinate system, so it is convenient to convert it to an earthfixed coordinate system. Figure 4 shows the body-fixed and earth-fixed coordinate systems fixed to an ROV for submarine operations. ${ }^{(12)}$ In navigation, the IMU is important and provides fast acceleration and angle data, but the accumulated error increases with time even when using a very expensive analog IMU. The USBL generally obtains position information and the position error does not increase with time. However, its interval is usually over 2 to 10 such that the error of position data occurs during the interval. For this reason, we devised the 


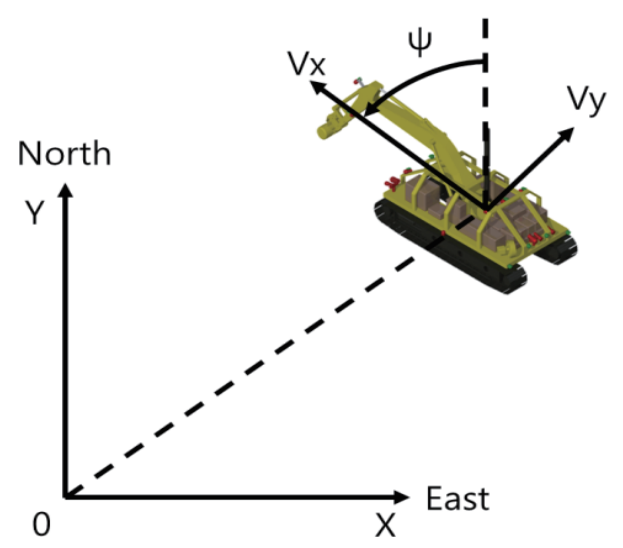

Fig. 4. (Color online) Relationship between ROV and global coordinates.

HNA using the IMU and USBL. By using the IMU data, the intermediate data of the USBL are compensated. However, in the real application of the HNA using the IMU and USBL, there exist sensor errors due to noises and disturbances in underwater environments. Thus, we developed the CHNA using the KF to more accurately estimate the location of the URI-R using hybrid RLG and USBL sensors by filtering out the noises and disturbances.

The frame used in the INS is the local-level frame (LLF), and the navigation equation used is $^{(13)}$

$$
\left[\begin{array}{c}
\dot{r}^{l} \\
\dot{v}^{l} \\
\dot{R}_{b}^{l}
\end{array}\right]=\left[\begin{array}{c}
D^{-1} v^{l} \\
R_{b}^{l} f^{b}-\left(2 \Omega_{i e}^{l}+\Omega_{e l}^{l}\right) v^{l}+g^{l} \\
R_{b}^{l}\left(\Omega_{i b}^{b}-\Omega_{i l}^{b}\right)
\end{array}\right],
$$

where $\boldsymbol{r}$ is the position vector, $\boldsymbol{v}$ is the velocity vector, $R$ is the Euler angle, $g$ is the local gravity vector, $f^{b}$ is the acceleration received from the IMU, and $\Omega_{i b}^{b}$ is the skew-symmetric matrix of the rotational velocity from the gyro.

$$
\begin{gathered}
\dot{r}^{l}=\left[\begin{array}{c}
\dot{\varphi} \\
\dot{\lambda} \\
\dot{h}
\end{array}\right]=\left[\begin{array}{ccc}
0 & \frac{1}{R_{M}+h} & 0 \\
\frac{1}{\left(R_{N}+h\right) \cos \varphi} & 0 & 0 \\
0 & 0 & 1
\end{array}\right]\left[\begin{array}{c}
v_{e} \\
v_{n} \\
v_{u}
\end{array}\right] \\
R_{M}=\frac{a\left(1-e^{2}\right)}{\left(\sqrt{1-e^{2} \sin ^{2}(\varphi)}\right)^{3}}
\end{gathered}
$$




$$
R_{N}=\frac{a}{\left(\sqrt{1-e^{2} \sin ^{2}(\varphi)}\right)^{3}}
$$

Here, $R_{M}$ is the radius of curvature of the meridian, $R_{N}$ is the radius of curvature, $h$ is the height of the USBL, $\varphi$ is the latitude of the USBL, $\lambda$ is the longitude of the USBL, $a$ is the radius at the equator, and $e$ is the eccentricity of the ellipsoid. ${ }^{(14)}$

$$
g^{l}=\left[\begin{array}{lll}
0 & 0 & -g
\end{array}\right]^{\mathrm{T}}
$$

$$
\underbrace{\omega_{i e}^{l}=\left[\begin{array}{c}
0 \\
\omega^{e} \cos \varphi \\
\omega^{e} \sin \varphi
\end{array}\right]}_{\text {vector }}, \underbrace{\Omega_{i e}^{\ell}=\left[\begin{array}{ccc}
0 & -\omega^{e} \sin \varphi & \omega^{e} \cos \varphi \\
\omega^{e} \sin \varphi & 0 & 0 \\
-\omega^{e} \cos \varphi & 0 & 0
\end{array}\right]}_{\text {skew-symmetric matrix }}
$$

$$
\underbrace{\omega_{e l}^{l}=\left[\begin{array}{c}
-\dot{\varphi} \\
\dot{\lambda} \cos \varphi \\
\dot{\lambda} \sin \varphi
\end{array}\right]=\left[\begin{array}{c}
-\frac{v_{n}}{R_{M}+h} \\
\frac{v_{e}}{R_{N}+h} \\
\frac{v_{e} \tan \varphi}{R_{N}+h}
\end{array}\right]}_{\text {vector }} \underbrace{\Omega_{e l}^{\ell}=\left[\begin{array}{ccc}
0 & -\frac{v_{e} \tan \varphi}{R_{N}+h} & \frac{v_{e}}{R_{N}+h} \\
\frac{v_{e} \tan \varphi}{R_{N}+h} & 0 & \frac{v_{n}}{R_{M}+h} \\
-\frac{v_{e}}{R_{N}+h}-\frac{v_{n}}{R_{M}+h} & 0
\end{array}\right]}_{\text {skew-symmetric matrix }}
$$

$$
\underbrace{\omega_{i b}^{b}=\left[\begin{array}{c}
\omega_{x} \\
\omega_{y} \\
\omega_{z}
\end{array}\right]}_{\text {vector }}, \underbrace{\Omega_{i b}^{b}=\left[\begin{array}{ccc}
0 & -\omega_{z} & \omega_{y} \\
\omega_{z} & 0 & -\omega_{x} \\
-\omega_{y} & \omega_{x} & 0
\end{array}\right]}_{\text {skew-symmetric matrix }}
$$

Here, $\omega_{x}, \omega_{y}$, or $\omega_{z}$ indicates the rotation speed of each axis and $\Omega_{i l}^{b}=R_{l}^{b}\left(\Omega_{i e}^{l}+\Omega_{e l}^{l}\right) R_{b}^{l}$.

In general, the nonlinear dynamic system is used as a first-order differential equation using the state equation. The error equation of the INS is derived and applied as

$$
\dot{x}^{l}=F^{l} x^{l}+G^{l} w,
$$

where $x$ is the vector of the internal parameters of the dynamic system that is referred to as the state vector, $F$ is called the dynamic matrix that propagates errors over time, $w$ is the random forcing function, assumed to be the Gaussian white noise associated with the inertial sensors, and $G$ is the noise distribution matrix. 


$$
\begin{gathered}
\dot{x}^{l}=\left[\begin{array}{ccccccccc}
\delta \varphi & \delta \lambda & \delta h & \delta v_{e} & \delta v_{n} & \delta v_{u} & \delta p & \delta r & \delta y
\end{array}\right]^{\mathrm{T}} \\
F^{l}=\left[\begin{array}{cccccccccc}
0 & 0 & 0 & 0 & \frac{1}{R_{M}+h} & 0 & 0 & 0 & 0 \\
0 & 0 & 0 & \frac{1}{\left(R_{N}+h\right) \cos \varphi} & 0 & 0 & 0 & 0 & 0 \\
0 & 0 & 0 & 0 & 0 & 1 & 0 & 0 & 0 \\
0 & 0 & 0 & 0 & 0 & 0 & 0 & f_{u} & -f_{n} \\
0 & 0 & 0 & 0 & 0 & 0 & -f_{u} & 0 & f_{e} \\
0 & 0 & 0 & 0 & 0 & 0 & f_{n} & -f_{e} & 0 \\
0 & 0 & 0 & 0 & 1 & 0 & 0 & 0 & 0 \\
0 & 0 & 0 & -\frac{1}{R_{N}+h} & 0 & 0 & 0 & 0 & 0 \\
0 & 0 & 0 & -\frac{\tan \varphi}{R_{N}+h} & 0 & 0 & 0 & 0 & 0
\end{array}\right]
\end{gathered}
$$

It is set to $G^{l}=w=0$.

The KF can be divided mainly into a prediction step and a correction step. ${ }^{(15-17)}$ In the prediction step, the following equations are used:

$$
\begin{gathered}
\hat{x}_{k}(-)=\Phi_{k \mid k-1} x_{k-1}(+), \\
P_{k}(-)=\Phi_{k \mid k-1} P_{k-1}(+) \Phi_{k \mid k-1}^{\mathrm{T}}+G_{k-1} Q_{k-1} G_{k-1}^{\mathrm{T}},
\end{gathered}
$$

where $\bar{x}_{k}(-)$ is the estimated state variable, $\Phi$ is the state transformation matrix, $x$ is the state variable, $P(-)$ is the estimated state covariance, $G$ is the disturbance noise, and $Q$ is the process noise covariance.

Equations (14)-(16) are used for calibrating the predicted value using an externally measured source.

$$
\begin{gathered}
K_{k}=P_{k}(-) H_{k}^{\mathrm{T}}\left[H_{k} P_{k}(-) H_{k}^{\mathrm{T}}+R_{k}\right]^{-1} \\
\hat{x}_{k}(+)=\hat{x}_{k}(-)+K_{k}\left[z_{k}-H_{k} \hat{x}_{k}(-)\right] \\
P_{k}(+)=\left[I-K_{k} H_{k}\right] P_{k}(-)
\end{gathered}
$$


Here, $K$ is the Kalman gain, $H$ is the observation matrix, $R$ is the measurement noise covariance, and $z$ is the external measurement vector. $P, R$, and $Q$ are selected as shown in the equations

$$
\begin{gathered}
P=\operatorname{diag}\left\{\begin{array}{lllllllll}
10^{2} & 10^{2} & 1^{2} & 0.05^{2} & 0.05^{2} & 0.05^{2} & 1^{2} & 1^{2} & \left.1^{2}\right\} \\
R=\operatorname{diag}\left\{(0.1 d)^{2}\right. & (0.1 d)^{2} & 1^{2} & 0.2^{2} & 0.2^{2} & 0.2^{2} & (0.1)^{2} & (0.1)^{2} & \left.1^{2}\right\} \\
Q=\operatorname{diag}\left\{1^{2}\right. & 1^{2} & 1^{2} & 0.1^{2} & 0.1^{2} & 0.1^{2} & 1^{2} & 1^{2} & \left.1^{2}\right\}
\end{array}\right.
\end{gathered}
$$

\section{Experiment of CHNA}

In this study, two experiments were conducted to verify the performance of the proposed CHNA. First, the heading motion obtained using the CHNA was tested and the performance of the CHNA was confirmed on land using the small UTV. Second, a navigation experiment in an actual sea area was conducted by applying the CHNA to the URI-R, which is the target platform of this study.

\subsection{Heading experiment using UTV}

To confirm the heading estimation of the proposed CHNA, experiments were conducted on land using the UTV (Fig. 2). The estimated value of the heading motion experiment when moving along a square track of $10 \mathrm{~m}$ on one side using the UTV is shown in Fig. 5, where the dotted line indicates the desired path. As a result of three times of rotation tracking test, the target was observed to converge to 90,180 , and $270^{\circ}$ at the initial $0^{\circ}$. The observed slightly large error is due to the rotation at the corner along the target heading in Fig. 5, and the estimated small convergence error to the target heading is due to an uneven track floor.

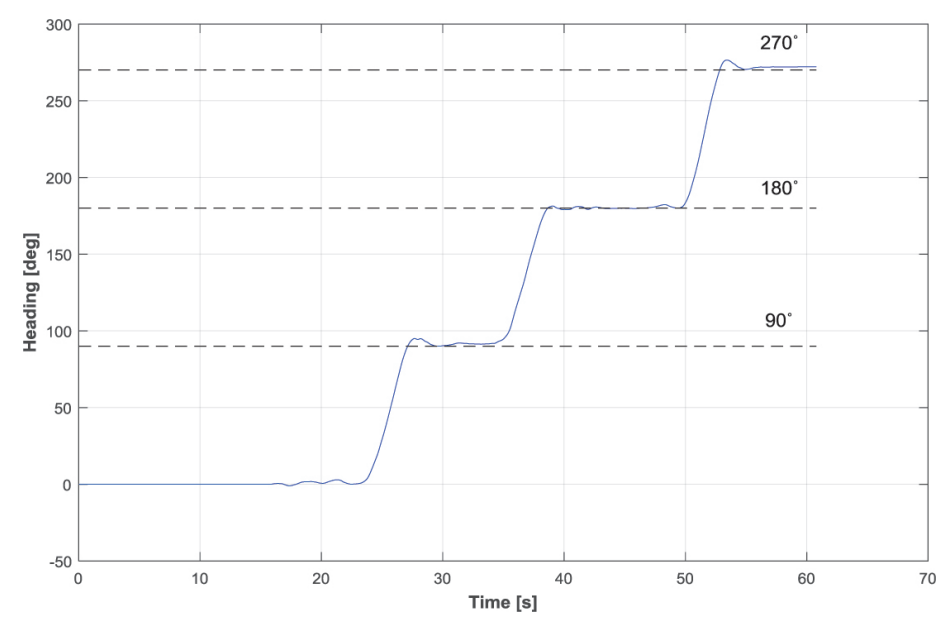

Fig. 5. Result of rectangle trajectory experiment. 


\subsection{Navigation experiment using URI-R}

To verify the performance of the designed CHNA, the trenching and navigation experiments of the URI-R were carried out in August 2018 in the East Sea. The latitude and longitude coordinates of the experimental area were $36^{\circ} 05^{\prime} 53.8^{\prime \prime} \mathrm{N}$ and $129^{\circ} 26^{\prime} 59.8^{\prime \prime} \mathrm{E}$, and the depth was $23 \mathrm{~m}$. Figure 6 shows the launching of the URI-R to the sea area for navigation experiment.

First, Fig. 7(a) shows the USBL acquisition signal sequence number on the abscissa each time the USBL signal is updated during the experiment, and the vertical axis indicates the time interval of the acquisition signal. Figure 7(b) shows a histogram showing the frequency of the sample time interval and the vertical axis as a log scale. The data update period of the USBL was set to $1 \mathrm{~s}$, and the experimental result shows that the update cycle of more than half a data is about $1 \mathrm{~s}$. The time to update the data was increased up to about $5 \mathrm{~s}$, but there was no case in which an invalid signal was measured. If the data update time exceeds $1 \mathrm{~s}$, it is considered that the transponder of the USBL is affected by the vibration caused by the platform performing the

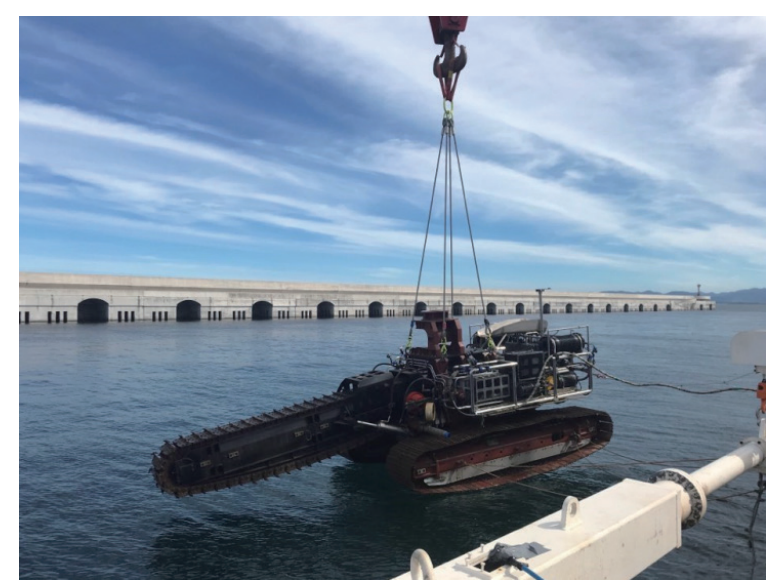

Fig. 6. (Color online) Picture of field experiment.

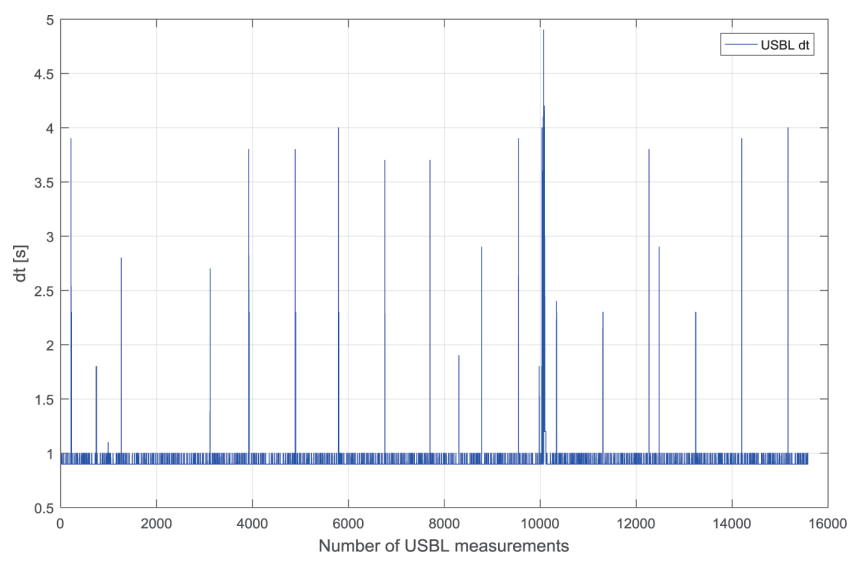

(a)

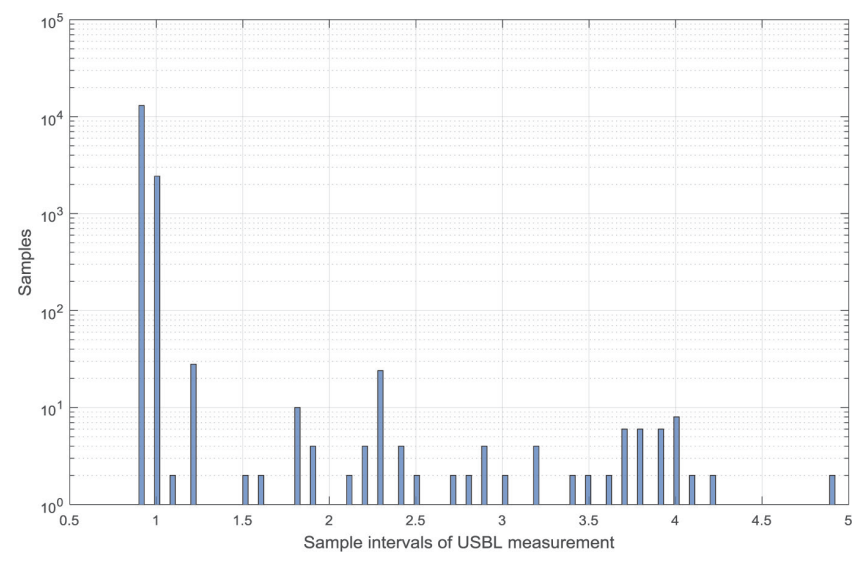

(b)

Fig. 7. (Color online) Sample intervals of USBL measurements of navigation experiment. (a) Sample intervals of USBL measurements and (b) their histogram. 
trenching operation or passing through an uneven ground. Because the depth of the experiment was not as large as $23 \mathrm{~m}$, it is considered that the data update was performed well.

The results of using the CHNA are shown in Fig. 8. Figure 8 shows the horizontal plane trajectory on which the URI-R has moved, and the USBL measurement position data are indicated by a dotted line and the navigation estimation position data obtained using the CHNA are indicated by a solid line. The navigation test was performed for about $4 \mathrm{~h}$ until the platform was launched and recovered at the same time as it was started. The platform was launched in a circular position, moved clockwise, and then retrieved from the square position after the experiment. At the beginning of the experiment, the experiment was conducted to move to the desired point. This experiment runs straight between the blue diamonds in Fig. 8. The heading of the URI-R was 30 degrees and moved about $29 \mathrm{~m}$. The error between the navigation result and the target position $[28.5,1.5]$ was measured as $[0.6,0.3]$.

Experimental results show that there are six densely populated parts in the data, which is due to the vibration of the platform during trenching. In the tests, the maximum speeds of the URI-R along the axes are $0.3228,0.3944$, and 0.1376 , and the movement speed of the platform is rather low.

Figure 9(a) shows that the results in Fig. 8 indicate the enlargement of the upper right [40, 12]. The A region has a denser shape owing to the larger position error due to the vibration generated by the tangential movement of the platform. This vibration also causes severe disturbances to the INS. Tracking errors in the $\mathrm{B}$ and $\mathrm{C}$ regions show relatively larger tracking errors that occurred owing to the bad signal state of the USBL, but it is shown that the application of the CHNA reduces the tracking errors and the smoothing of the trajectory tracking. Zigzag-like movement paths, such as in the $\mathrm{B}$ and $\mathrm{C}$ regions are forms of movement that cannot be achieved with a large, heavy URI-R. The proposed navigation algorithm CHNA shows the result of compensating the tracking errors compared with the measurement using only the USBL sensor. Next, Fig. 9(b) shows that the results in Fig. 8 indicate the enlargement of the right center $[35,-6]$. Data in the A region show that there are position errors in the USBL

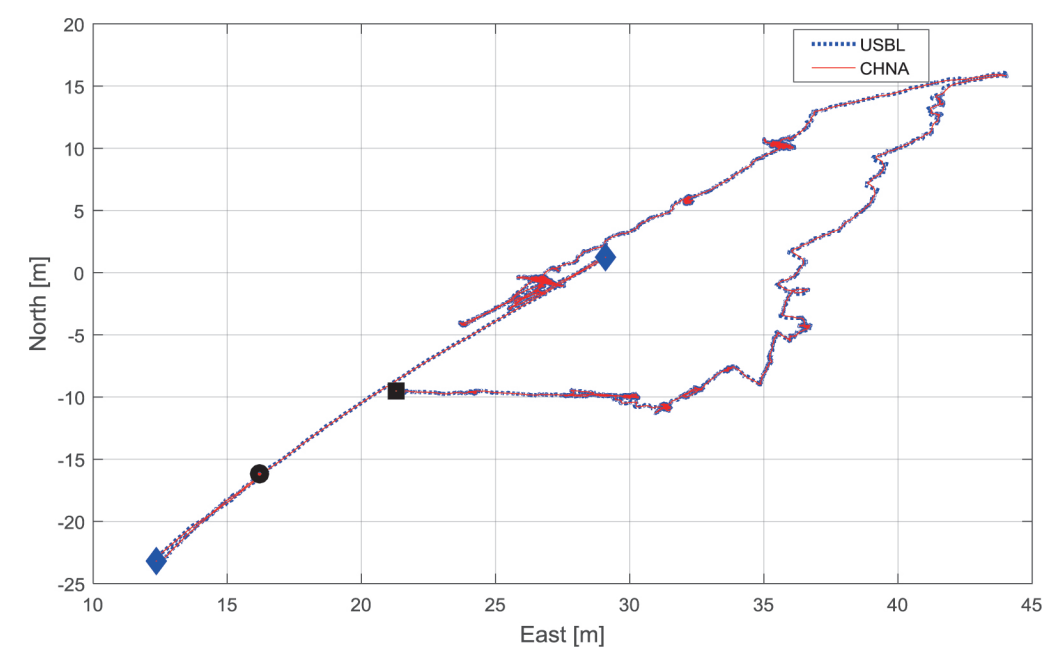

Fig. 8. (Color online) $X-Y$ plane trajectories \#1. 


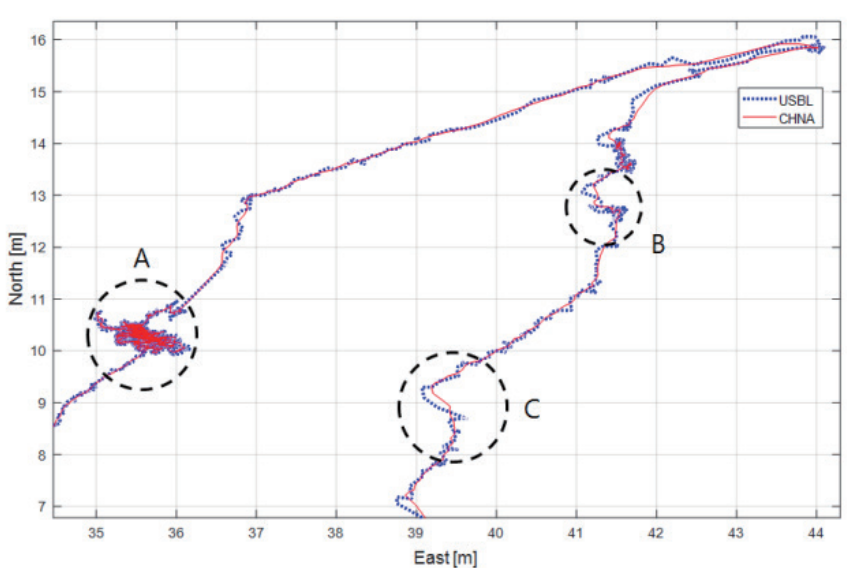

(a)

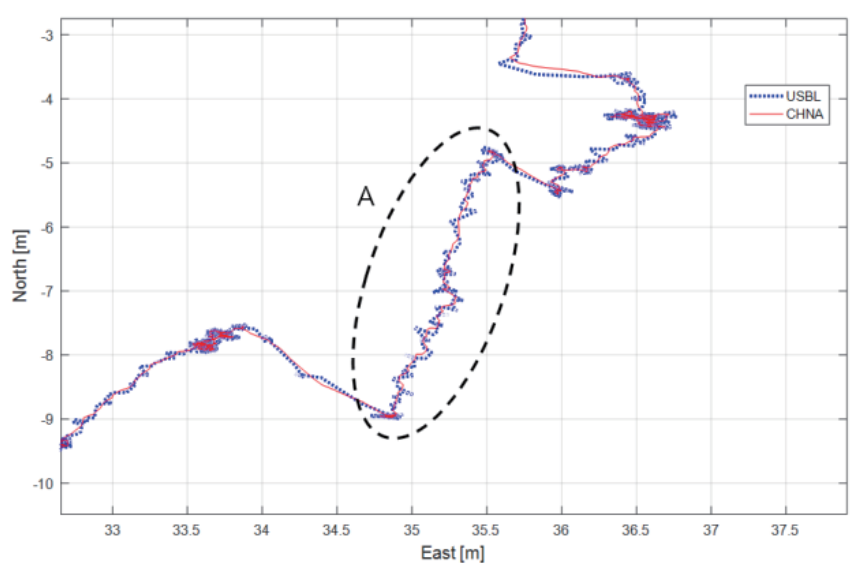

(b)

Fig. 9. (Color online) $X-Y$ plane trajectories \#2. (a) Top right and (b) center right.
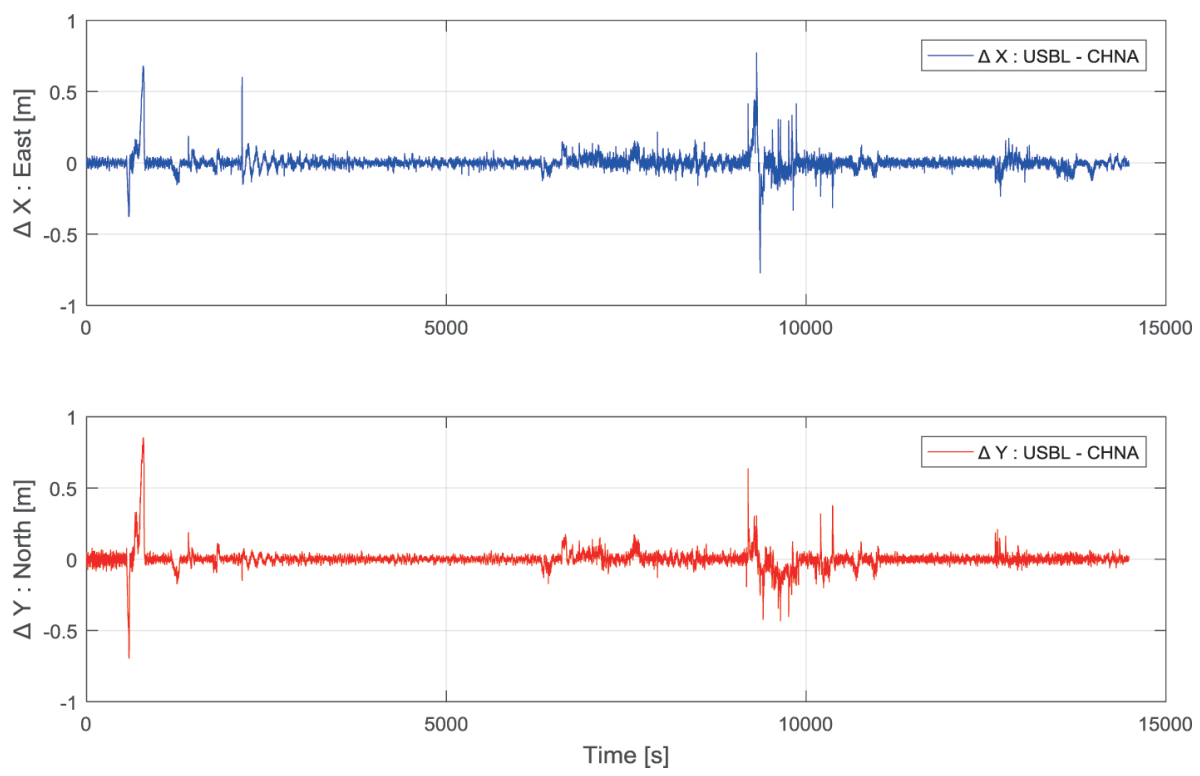

Fig. 10. (Color online) Difference between position estimates obtained using only USBL and CHNA.

signal and that the position data are unsatisfactory. However, the proposed CHNA results show that these errors are reduced.

Figure 10 shows the position estimation difference of the navigation between the measurement using only the USBL and the CHNA. Even under the very low movement speed of the platform and the absence of USBL blackout, the maximum difference between the measurement using only the USBL and the CHNA is shown to be less than $1 \mathrm{~m}$. The part where the difference increases is considered as the time when the platform performs the trenching operation. Through the experimental results, the good performance of the developed CHNA is verified. 


\section{Conclusions}

In navigation, an IMU is important because it provides fast acceleration and angle data, but the accumulated error increases with time. A USBL generally obtains position information and the position error does not increase with time. However, its interval is usually over 2 to $10 \mathrm{~s}$ such that the position error occurs during the interval. On the basis of this result, we developed a CHNA based on a Kalman filter to more accurately estimate the location of the URI-R using hybrid INS (IMU), RLG, and USBL sensors by filtering out noises and disturbances.

To apply the CHNA to a real underwater trenching vehicle called URI-R, we developed a small UTV as a test bed to test the performance of the CHNA. Then, we applied the CHNA to the URI-R and verified the superior performance of trajectory tracking through a real underwater test.

To verify the performance of the proposed algorithm, we analyzed the performance of the CHNA utilizing the data of the RLG, IMU, and USBL obtained from the UTV and URI-R land/ sea experiments. Experimental results show that the maximum position difference between the measurement using only the USBL and the CHNA is about $1 \mathrm{~m}$. From the data of the navigation experiments, it was confirmed that the developed CHNA could follow the desired position and attitude better than the case of using only the USBL. Through the experimental results, the good performance of the developed CHNA is verified.

\section{Acknowledgments}

This research is part of the project of the National Research Foundation of Korea (NRF2016R1A2B4011875) and the project entitled "R\&D center for underwater construction robotics", South Korea (PJT200539), funded by the Ministry of Oceans and Fisheries (MOF).

\section{References}

1 P. M. Lee, B. H. Jeon, S. M. Kim, H. T. Choi, C. M. Lee, T. Aoki, and T. Hyakudome: Oceans'04, MTTS/IEEE TECHNO-OCEAN'04 3 (2004) 1586.

2 W. Somers: Ph.D. Thesis, New Brunswick (2011).

3 K. R. Britting: Inertial Navigation System Analysis (Wiley Interscience, New York, 1971).

4 J. Bijker and W. Steyn: Control Eng. Pract. 16 (2008) 1509.

5 J. Wendel and G. F. Trommer: Aerosp. Sci. Technol. 8 (2004) 627.

6 P. Ridao, D. Ribas, E. Hernandez, and A Rusu: 2011 IEEE Int. Conf. Robotics and Automation (ICRA) (IEEE, 2011) 2344-2349.

7 D. Ribas, P. Ridao, A. Mallios, and N. Palomeras: 2012 IEEE Int. Conf. Robotics and Automation (ICRA) (IEEE, 2012) 4898-4903.

8 P. M. Lee, B. H. Jun, K. Kim, J. Lee, T. Aoki, and T. Hyakudome: J. Oceanic Eng. 32 (2007) 392.

9 P. Lee, B. Kim, H. Shim, H. Baek, S. Baek, J. Park, and B. Jun: Proc. Korean Society of Ocean Engineers (2015) 450.

10 D. W. Jung, S. M. Hong, J. H. Lee, H. J. Cho, H. S. Choi, and M. T. Vu: Proc. Eng. Technol. Innovation 9 (2018) 17.

11 Y. J. Lee, D. H. Choi, D. H. Ji, T. V. Mai, H. S. Choi, and J. Y. Kim: Proc. Korean Marine Robot Technology Society (2015) 84.

12 T. I. Fossen: Marine Control System (Marine Cybernetics, Trondheim, 2002).

13 A. Noureldin, T. B. Karamat, and J. Georgy: Fundamentals of Inertial Navigation, Satellite-based Positioning and Their Integration (Springer, Heidelberg, 2013). 
14 H. Ellingsen: Master's Thesis, Institutt for Teknisk Kybernetikk (2008).

15 M. Karimi, M. Bozorg, and A. R. Khayatian: 2013 First RSI/ISM Int. Conf. (ICRoM, 2013) 62-67.

16 P. Kim: CreateSpace Independent Publishing Platform (2011).

17 T. G. Kim, H. T. Choi, and N. Y. Ko: 13th Int. Conf. Control, Automation and Systems (2013) 195.

\section{About the Authors}
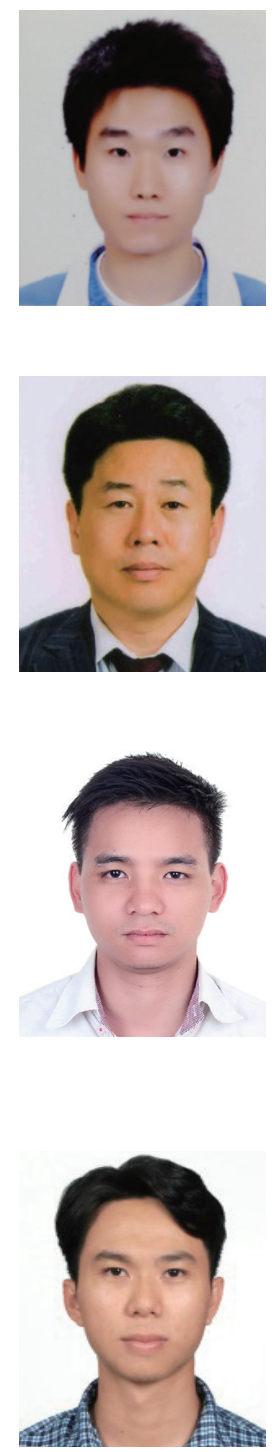

Ngoc Duc Nguyen received his B.S. degree from Ho Chi Minh University of Technology, Vietnam, in 2014 and his M.S. degree from Korea Maritime and Ocean University, Korea, in 2016. Since 2016, he has been a PhD candidate at Korea Maritime and Ocean University. His research interests are in the dynamics, control, and guidance of underwater vehicles.

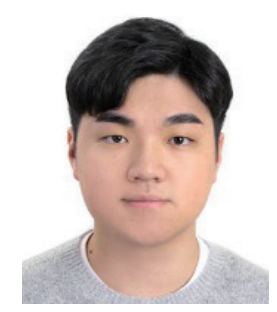

Seo-Kang Kim received his B.S. degree in Mechanical Engineering from Korea Maritime and Ocean University, Busan, Republic of Korea, in 2017. He is currently working toward his M.S. degree at the Korea Maritime and Ocean University Graduate School, Republic of Korea. His research interests include the design of a machine and structural analysis.

Dae-Hyeong Ji received his M.S. degree in Mechanical Engineering from Korea Maritime and Ocean University, Busan, Korea, in 2014. He is currently working toward a Ph.D. degree in the Department of Convergence Study on the Mechanical Engineering at Korea Maritime and Ocean University, Busan, Korea. His research interests include navigation and control systems, sensors, and intelligent control and robotics.

Hyeung-Sik Choi received his M.S. degree in Mechanical Engineering from South Carolina University, USA, in 1989 and his Ph.D. degree in Mechanical Engineering from North Carolina State University, USA, in 1993. Since 1997, he has been a professor at Korea Maritime and Ocean University. His research interests are in the design and control of ocean robotics, unmanned ocean systems, and underwater LED communication.

Mai The Vu received his M.S. degree in Mechanical Engineering from Korea Maritime and Ocean University, Busan, Korea, in 2015. He is currently working toward a Ph.D. degree in the Department of Convergence Study on the Ocean Science and Technology, School of Ocean and Technology, Korea Maritime and Ocean University, Busan, Korea. His research interests include modeling and control systems, optimal control, detection and estimation for navigation systems, and intelligent control and robotics. 